
cultures

Les cahiers de l'Acedle

15-1 | 2018

La conceptualisation grammaticale

\title{
La difficile adaptation de la didactique convergente au contexte arabophone
}

\section{Emna Souilah}

\section{OpenEdition}

Journals

Édition électronique

URL : http://journals.openedition.org/rdlc/2729

DOI : $10.4000 /$ rdlc. 2729

ISSN : 1958-5772

Éditeur

ACEDLE

Référence électronique

Emna Souilah, «La difficile adaptation de la didactique convergente au contexte arabophone », Recherches en didactique des langues et des cultures [En ligne], 15-1 | 2018, mis en ligne le 02 janvier 2018, consulté le 23 avril 2019. URL : http://journals.openedition.org/rdlc/2729; DOI : 10.4000/ rdlc. 2729

Ce document a été généré automatiquement le 23 avril 2019

\section{c) (i) $\Theta$}

Recherches en didactique des langues et des cultures is licensed under a Creative Commons AttributionNonCommercial-NoDerivatives 4.0 International License 


\title{
La difficile adaptation de la didactique convergente au contexte arabophone
}

\author{
Emna SOUILAH
}

1 Les efforts déployés pour optimiser l'apprentissage du français à travers le monde s'appuient sur des travaux de recherche dans le domaine de la didactique des langues, qui ciblent aujourd'hui des contextes locaux. Ces travaux visent à améliorer concrètement la qualité de l'enseignement de cette langue dans ces contextes, à aplanir les difficultés spécifiques rencontrées par les apprenants et à proposer des moyens efficaces pour $\mathrm{y}$ parvenir.

Dans le contexte maghrébin, les alternatives méthodologiques ${ }^{1}$ adoptées jusque-là, dont la plupart sont conçues dans et pour d'autres contextes, s'avèrent inefficientes (Souilah, 2014 ; Bouhdiba, 2014). Elles montrent que l'enjeu actuel de toute réforme est de trouver des solutions aux problèmes posés à l'enseignement-apprentissage du français qui émanent du contexte lui-même ou qui lui sont initialement adaptées.

3 Dans cette optique, la didactique convergente semble être prometteuse, car elle se présente comme une démarche porteuse de solutions à des problèmes spécifiques à chaque contexte. Élaborée en Europe, elle se veut susceptible d'adaptation à n'importe quel contexte d'enseignement-apprentissage de la langue, moyennant des réajustements ciblés.

4 Cette didactique s'appuie sur le potentiel cognitif des apprenants et sur leur patrimoine linguistique. Elle articule les composants de leur répertoire bilingue pour concevoir des approches méthodologiques adaptées. À ce titre, elle réinvestit les acquis de la linguistique contrastive (LC) pour une comparaison ciblée des langues en présence lors de l'apprentissage.

5 Aujourd'hui, dans le monde arabophone, les chercheurs en didactique des langues, conscients de l'importance de cette démarche, ont procédé à son adaptation à ce contexte 
et à l'élaboration du Guide du formateur d'enseignants en didactique convergente (françaisarabe) (OIF : 2009).

Dans cette perspective, les questions qui se posent sont de deux ordres. Elles sont, d'un côté, liées à la spécificité de la version de la didactique convergente destinée au monde arabophone: est-elle concrètement porteuse de solutions? A-t-elle pu réellement contourner la faiblesse du courant dont elle est, dans une certaine mesure, issue ? D'un autre côté, ces questions ont trait aux terrains où l'on se propose de l'implanter: la didactique convergente est-elle possible dans les contextes tunisien et algérien, par exemple, qui ont toujours fonctionné avec des méthodologies conçues pour d'autres contextes, que les autorités locales n'ont pas réussi à adapter?

Il semblerait qu'en l'état actuel des choses elle ait encore un long chemin à faire et ce pour les raisons que nous nous proposons d'analyser dans cet article.

Dans notre analyse, nous nous appuyons sur le guide arabophone numérique de l'Organisation internationale de la francophonie, élaboré par un réseau de didacticiens du monde arabe (Miled, 2010a : 44) ${ }^{2}$. Ce document est destiné aux formateurs ayant la charge de former les enseignants de français en didactique convergente (DC), dans les pays où le français a le statut de langue seconde. Il présente le cadre théorique et conceptuel de cette didactique et propose pour les sessions de formation des enseignants des fiches pratiques susceptibles d'adaptation à chacun de ces pays.

9 Nous aborderons, dans le présent article, trois points complémentaires. Nous donnerons un bref aperçu de la DC dans la littérature scientifique. Nous présenterons la version adaptée au monde arabe. Nous finirons par une étude critique de la fiche syntaxique sur la reprise pronominale.

\section{La didactique convergente dans la littérature scientifique}

10 Les travaux du symposium organisé par le Conseil de l'Europe sur les liens entre l'enseignement de la langue maternelle et l'enseignement des langues vivantes (1973) ont mis l'accent sur la nécessité d'envisager de façon intégrée l'enseignement des langues. Dans cette visée, les réflexions de Roulet (1980) sur l'intérêt d'une didactique intégrée des langues maternelles et secondes ont posé les jalons d'une adaptation de cette démarche à tous les contextes d'enseignement de la langue. Dabène a fourni des indications encore plus précises concernant le décloisonnement des enseignements linguistiques (Dabène, 1992 : 13). Plus récemment, le Cadre européen commun de référence pour les langues a défini des orientations au sujet de cette démarche (CECRL, 2000 : 129). Sur le plan pratique, cette démarche a été mise en place d'une manière réussie dans l'enseignement du français à l'école moyenne bi/plurilingue au Val d'Aoste où les programmes stipulent qu'il importe [...] d'exploiter le patrimoine linguistique que l'élève a acquis et vit dans son expérience familiale et sociale (Cavalli, $2000: 2$ ).

11 Dans son évolution, la DC s'est continuellement appuyée sur des principes construits sur la base de la pensée de Vygotsky qui en constitue le fondement psycholinguistique et une référence incontournable. Plusieurs travaux effectués dans le domaine de l'acquisition des langues sont basés sur l'idée que lorsque l'enfant assimile une langue étrangère, il dispose déjà dans sa langue maternelle d'un système de significations qu'il transfère dans l'autre langue (Vygotsky, $1934:$ 374-376). 
12 Ainsi, pour permettre à l'apprenant de recevoir une formation linguistique cohérente, la DC prend appui sur ces langues maternelles des élèves qui ne sont pas [...] langues de l'école pour les orienter vers la voie de la réflexion linguistique qui mène à une conscience métalinguistique approfondie (Cavalli, 1994: 19).Dans ce cadre, la question est de savoir quels sont les réajustements effectués sur cette didactique pour en faire une démarche commune à une diversité de contextes arabophones locaux.

\section{La didactique convergente dans le guide de l'OIF}

13 La $\mathrm{DC}^{3}$ se présente dans ce document comme une démarche contextualisée qui, pour concevoir ses méthodes d'enseignement $d u$ français, s'appuie sur une description sociolinguistique préalable et sur l'analyse des difficultés réelles rencontrées par les apprenants. Elle propose de trouver des solutions de remédiation aux difficultés posées à l'apprentissage de cette langue en tenant compte du passé linguistique de l'apprenant. Elle fonde ses travaux sur des études comparatives des systèmes linguistiques en présence et vise le rapprochement des deux didactiques de l'arabe et du français (Miled, 2010a : 48). Ses principales entrées sont d'ordre institutionnel et pédagogique. La première entrée est du ressort des concepteurs de programmes. Il s'agit de procéder à un rapprochement linguistique et méthodologique en vue d'une harmonisation globale des curricula relatifs à l'enseignement des deux langues. La deuxième concerne la préparation des séquences didactiques qui s'appuiera sur des repères méthodologiques fournis par la DC.

Le premier examen du guide de l'OIF a révélé des zones d'ombre que nous allons élucider en mettant en lumière la spécificité sociolinguistique du contexte maghrébin.

\section{La spécificité sociolinguistique du contexte maghrébin}

15 Le transfert de la didactique convergente au monde arabophone implique des adaptations et des réajustements liés à sa spécificité sociolinguistique. Pour une contextualisation efficace, il faut comprendre le plus complètement et le plus profondément possible les paramètres locaux. On doit aller jusqu'au niveau "microscopique» de tel groupe d'apprenants dans tel établissement à tel endroit (Blanchet, 2009 : 2).

Les études sociolinguistiques de Taleb-Ibrahimi (2010) et de Brahim (1994) sont éclairantes quant aux contextes tunisien et algérien. Ces contextes se caractérisent par leur complexité sociolinguistique en raison de la question non élucidée de la langue maternelle dans son rapport avec l'arabe littéral et de la variété de la langue arabe: l'arabe du texte coranique, celui de la littérature classique, l'arabe standard, qu'on appelle aussi l'arabe moderne, les différents parlers qui varient d'un pays arabe à l'autre et qui ne sont pas forcément compris par tous les arabophones. Ces parlers varient également à l'intérieur d'un même pays.

17 Ces études soulignent que la première scolarisation de l'enfant est marquée par la rupture brutale avec sa langue maternelle. Il est directement mis en contact avec l'arabe littéral qui est :

un artefact de langue n'ayant aucune existence réelle dans les pratiques de tous les locuteurs arabophones [...] et qui [...] $\mathrm{m}[\mathrm{et}]$ en opposition la langue de la maison et la langue de l'école, la langue de la mère et la langue de la maitresse (TalebIbrahimi, $2010: 40$ ).

[On] lui inculque souvent un ensemble figé de normes (Brahim, 1994 : 14). 

terminologie équivoque, imprécise et parfois inadéquate où l'arabe est désigné par les expressions langue maternelle et langue première. On peut lire :

la didactique convergente du français langue seconde et de l'arabe, langue première (OIF, 2009:5) ;

l'expérience grammaticale déjà intériorisée en L1 (ibid. : 11) ;

la langue maternelle constitue le fondement psychomoteur (ibid.) ;

Constat d'un recours [...] à l'arabe : la tentation est d'utiliser la langue première (ibid. : 12);

le recours à la langue maternelle (ibid. : 13).

À la fin du guide, le mot arabe prend un autre sens :

l'arabe est la langue où l'élève apprend à écrire, à calculer et à accéder à l'ensemble des disciplines scolaires (ibid. : 12).

Aussi, sur la base des définitions données à langue première et langue maternelle (Cuq, 2006) , l'arabe enseigné à l'école dans ces contextes n'est-il ni langue première ni langue maternelle. La langue maternelle est définie comme étant :

la langue acquise la première par le sujet parlant dans un contexte où elle est aussi la langue de communication (ibid.)

et plus loin :

la complexité de la dénomination de langue maternelle amène à lui substituer [...] langue première ou L1 (ibid.).

De ce fait, la démarche adaptée au monde arabophone est une didactique convergente du français et de l'arabe enseigné à l'école. Elle est en totale concordance avec son fondement psycholinguistique fondé sur le principe constructiviste qui « recommande de partir du connu ou de l'acquis chez l'élève » (OIF, 2009 : 11).

Il semble ainsi que ce projet de l'OIF de concevoir une didactique convergente commune à de nombreux pays arabes s'écarte d'une démarche contextualisée soucieuse des spécificités sociolinguistiques des contextes locaux pour se rapprocher d'une approche comparatiste et contrastive de deux langues enseignées à l'école. Dans cette optique, les 
fiches proposées pour la formation des enseignants de français en DC semblent s'inspirer de la linguistique contrastive. Une comparaison rapide des deux démarches permettra de mesurer l'écart entre l'une et l'autre.

\section{L'analyse contrastive et la didactique convergente}

L'AC s'appuie sur l'idée que la confrontation structurelle des langues en contact permet de prévenir le phénomène d'interférence linguistique « indépendamment des réalisations des apprenants » (Marquilló Larruy, 2006 : 66). Cette hypothèse, critiquée et invalidée, a permis à ce courant d'évoluer vers l'analyse d'erreurs qui consiste à étudier les erreurs interférentielles des apprenants pour mieux comprendre leurs mécanismes. Ce courant a aussi présenté des faiblesses provenant de « la confusion entre la description linguistique d'une structure déterminée et la manière ou les modalités mises en œuvre par un apprenant pour se les approprier» (Marquilló Larruy, 2006 : 66) : ce qui est en contact, lors de l'apprentissage d'une langue étrangère, ce ne sont pas les systèmes linguistiques des langues source et cible mais la grammaire intériorisée de la langue maternelle de l'apprenant et ce qu'il apprend de la nouvelle langue. C'est ainsi que le domaine de traitement des erreurs a évolué d'une part vers la recherche sur l'interlangue dont l'objectif est de « décrire les grammaires intériorisées à travers les activités langagières qui les manifestent » (Porquier et Besse, $1984: 216)$ et d'autre part vers les travaux sur les parlers bilingues.

Cet aperçu rapide sur l'évolution du domaine de traitement des erreurs appelle deux remarques. La première est relative à l'importance accordée au rôle joué par les langues maternelles dans la production des erreurs. La seconde remarque est liée aux défaillances méthodologiques en rapport avec l'identification et l'interprétation des erreurs, que présente chaque courant.

À la question de savoir où se positionne la DC par rapport à ces courants, Miled souligne que cette démarche «n'est pas en rupture radicale avec la linguistique contrastive, dont elle réinvestit les acquis » (Miled, 2010a:5), mais qu'elle s'en écarte par l'élargissement de son domaine d'application qui s'ouvre sur des questions discursives et culturelles. De plus, la DC ne se limite pas aux descriptions inspirées de l'AC : elle se propose de chercher plus finement l'origine des erreurs des apprenants (OIF, 2009:9). Elle marque aussi sa singularité en reprochant à ce courant de ne pas soulever la question de l'appropriation par l'apprenant de ces deux langues (OIF, 2009: 13) et se fixe comme objectif « d'expliciter le trajet de l'élève dans son passage de L1 à L2, afin d'élucider l'activité et le processus d'apprentissage » (Miled, 2010a: 5). Enfin, on considère aujourd'hui que cette «nouvelle » démarche didactique a la particularité de travailler sur la remédiation et propose des solutions adéquates.

Pour examiner la réalisation didactique de la DC dans le guide, nous allons parcourir les fiches pratiques et étudier la fiche syntaxique sur la reprise pronominale. 


\section{Étude de la réalisation didactique de la DC à partir des fiches pratiques}

Ce guide propose des fiches pratiques dédiées à la formation des enseignants dans des domaines variés. Chaque fiche a deux orientations :

- une orientation linguistique (comparaison d'un fait de langue en référence aux systèmes linguistiques de la L1 et de la L2) ;

- une orientation didactique (la manière dont s'effectue l'enseignement de ce fait de langue en L1 et en L2) débouchant sur des propositions d'activités à présenter aux enseignants en formation.

Toutes les fiches sont élaborées selon la même démarche : elles définissent la compétence visée par cette formation, présentent ses objectifs spécifiques et proposent un ensemble d'erreurs « interférentielles » récurrentes chez les apprenants arabophones. Dans chaque fiche, l'interprétation des erreurs est précédée d'une comparaison des faits linguistiques dans les deux langues, l'arabe littéral et le français.

31 Ces fiches présentent quelques limites qui semblent compromettre la démarche envisagée. Certaines sont d'ordre méthodologique et concernent l'ensemble des fiches, les autres sont exclusives à la fiche portant sur la reprise pronominale et liées aux connaissances grammaticales.

\section{Les problèmes d’ordre méthodologique}

Dans toutes les fiches de formation, l'entrée d'ordre institutionnel, préconisée comme un premier moyen d'aborder l'élaboration des fiches de formation est absente : les curricula dans les deux langues ne sont pas pris en considération et les méthodes pédagogiques ne sont pas prescrites. De ce fait, le passage direct à la préparation des séquences didactiques engendre les problèmes suivants :

- absence de convergence méthodologique : l'orientation didactique des fiches ne tient pas compte de la manière dont s'effectue l'enseignement des faits de langue en arabe ;

- les objectifs spécifiques sont uniquement relatifs à la classe de français ;

- les activités proposées sont loin de s'appuyer sur une conception de la grammaire qui suppose que les démarches dans l'enseignement de l'arabe et du français visent une action coopérative. Cette action implique que des modalités d'apprentissage soient intégrées dans le cadre d'une approche commune appelant des stratégies à mettre en pratique conjointement dans ces enseignements.

De surcroit, ces fiches présentent un ensemble d'erreurs sans préciser à quel stade de l'apprentissage elles sont produites (présystémique, systémique, postsystémique) (Corder, 1980 : 13). Il s'agit de distinguer ce qu'on appelle les erreurs logiques, celles commises avant l'apprentissage d'un fait de langue de celles commises après. Ces erreurs sont systématiquement attribuées au transfert négatif de la langue source sans prise en compte de la complexité et de la diversité des causes potentielles de ces erreurs (Porquier, 1994 : 207). De plus, elles sont classifiées par rapport à un seul des trois repères utilisés dans l'analyse d'erreurs, malgré l'importance des deux autres.

1. Le système de la langue étrangère, c'est-à-dire celui des natifs, ce qui implique des jugements de norme (description linguistique) 
2. L'exposition antérieure à la langue étrangère, c'est-à-dire qui [...] a été déjà été étudié et est présumé appris (description pédagogique)

3. La grammaire intériorisée d'un apprenant à un stade donné et dans son développement longitudinal (Porquier, 1991 : 213). origine et sont susceptibles au moins d'une double interprétation. Nous en présentons quelques exemples qui illustrent cette complexité. Dans le guide, un tableau comparatif propose des phrases fautives en français et leurs correspondances en arabe dont la structure est supposée être à l'origine de ces erreurs.

1. Ali a eu faim. Sa mère a donné-lui une pomme.

2. Ali a eu faim. Sa mère a donné le une pomme.

3. Alia a eu faim. Sa mère a donné à elle une pomme (OIF, 2009 : 27). la fiche sur la pronominalisation. Cette fiche présente ainsi la règle de la position des pronoms personnels par rapport au verbe de la phrase affirmative.

Place du pronom conjoint autre que le sujet :

Le verbe est à un autre mode que l'impératif (affirmatif) et l'infinitif :

a. un seul pronom conjoint : il se met devant le verbe (devant l'auxiliaire si le verbe est à un temps composé) :

On le voit/qu'on lui obéisse. /Nous lui avons répondu.

b. Plusieurs pronoms conjoints : [...] Quand le verbe est précédé de deux pronoms personnels objets, l'un direct l'autre indirect, celui-là se place le premier : nous le lui dirons.

Le verbe est à l'impératif affirmatif

Si un des pronoms est de la 1ère ou 2ème personne, l'objet indirect prend la forme du pronom disjoint et se place ordinairement en seconde position : Passez-la-moi. Montre-les-moi. Dis-le-moi (OIF : 28).

Cette règle grammaticale appelle trois remarques relatives à trois points :

- Le premier point révèle l'omission totale de la catégorie des verbes ayant des constructions en «à lui/y », ceux qui admettent indifféremment les compléments animés et inanimés, versus les verbes suivis de « lui » qui privilégient les animés (Blanche-Benveniste, 1975). Ces verbes français admettant des pronoms postposés, précédés d'une préposition (comme penser à lui, renoncer à lui, etc.) sont susceptibles de favoriser des erreurs intralinguales telles que celles qu'on considère comme des erreurs interférentielles. 
- Le second point est relatif à la place des pronoms les uns par rapport aux autres dans la phrase affirmative (voir : 1.b). Dans cette règle, seuls les pronoms le et lui sont proposés en exemples. Or, si l'on prend le cas de tous les pronoms conjoints qui peuvent avoir les mêmes fonctions grammaticales, tels que me, te, se, nous, vous - comme dans la phrase suivante : Nous te $(\mathrm{CI})$ le $(\mathrm{CD})$ dirons - la position des pronoms s'inverse complètement et va à l'encontre de la règle.

- Le troisième point évoque également la position des pronoms les uns par rapport aux autres dans la phrase impérative (voir : 2). Or, quand le pronom CD est partitif, il se place après le $\mathrm{CI}$ et non avant lui : Donne-m'en un; donne-lui-en deux; etc. Cela aboutit à des constructions contraires à celles présentées dans cette fiche.

Enfin, dans le cadre de l'approche contrastive, le paragraphe sur le mode impératif ne propose pas d'exemples de phrases impératives arabes qui correspondent à celles des phrases françaises présentées dans la colonne gauche du tableau comparatif, mais des exemples de phrases affirmatives arabes contenant des noms indéfinis qui, transformés en pronoms en français, donnent des pronoms CD partitifs, tels que : il lui a donné un livre / Il lui en a donné un.

39 À ce niveau, le tableau abandonne la démarche comparative, pourtant indispensable et très éclairante, pour se limiter à des explications relatives aux fonctionnements syntaxiques spécifiques aux pronoms personnels de chaque langue.

\section{Conclusion}

L'examen du guide de l'OIF mène au constat d'un double décalage :

- entre la version initiale de la DC et la version adaptée au monde arabophone par la mise à l'écart des langues maternelles des apprenants. Cette nouvelle version ne semble pas être soucieuse des particularités sociopsycholinguistiques des contextes locaux et éloigne cette didactique d'une démarche réellement contextualisée ;

- entre les orientations théoriques de la DC et sa réalisation didactique dans les fiches pratiques. Ces fiches, destinées seulement à la formation des enseignants de français et qui n'incluent pas les enseignants d'arabe, ne prennent pas en considération les approches privilégiées dans l'enseignement des faits de langue dans la classe d'arabe. Il faut noter que les curricula des deux langues ne sont ni mentionnés ni comparés.

41 De plus, ces fiches montrent que, malgré l'écart qu'affiche la DC vis-à-vis de l'analyse contrastive, elle semble renouer avec ce courant et adopte sa démarche consistant à attribuer systématiquement certaines erreurs au transfert négatif de l'arabe. Il s'agit, de surcroit, de l'arabe enseigné à l'école.

42 La nouvelle version de la didactique convergente destinée au contexte arabophone montre encore une fois que les approches conçues en Europe, même si elles se veulent contextualisables, semblent résister à leur implantation dans les contextes qui ne présentent pas de particularités sociopsycholinguistiques similaires au contexte d'origine.

Ainsi, nous pensons que, pour optimiser l'apprentissage du français et améliorer la qualité de son enseignement dans le contexte arabophone, il serait utile de commencer par régler les problèmes que pose l'enseignement de l'arabe littéral dans son rapport avec les langues maternelles des apprenants dans les contextes locaux. 


\section{étrangères dans ce contexte, cette dernière soulignant que :}

si la langue arabe est bien enseignée comme une langue vivante et de grande culture, les élèves sauront transférer, au moment opportun, les compétences acquises dans leur apprentissage des autres langues (Taleb-Ibrahimi : 2010, 45).

\section{BIBLIOGRAPHIE}

Besse, H. ; Porquier, R. (1991). Grammaire et didactique des langues. Paris : Hatier.

Blanchet, Ph. (2009) «Contextualisation didactique » : de quoi parle-t-on ? Interview consultée en janvier 2014. http://eprints.aidenligne-francais-universite.auf.org/147/1/pdf.pdf.

Blanche-Benveniste, C. (1975). Recherche en vue d'une théorie de la grammaire française. Paris : Champion.

Brahim, A. (1994). Linguistique contrastive et fautes de français. Tunis : Publications de la faculté des lettres de la Manouba.

Bouhadiba, F. «L'approche par compétences en Algérie : entre mythe, réalité et défis » in Babault, S. et al. (dir.) Actes du colloque international « Contexte global, contextes locaux, tensions, convergences, enjeux en didactique des langues ». Paris : FIPF, p. 83-98.

Cadre européen commun de référence pour les langues : apprendre, enseigner, évaluer (CECRL).

Cavalli, M. (2005). Education bilingue et plurilinguisme. Le cas du Val d'Aoste. Paris : Didier-CREDIF, coll. LAL.

Cavalli, M. (1994). «L'enseignement des langues : vers une didactique intégrée », L'école valdôtaine , n 24, p. $18-22$.

Corder, P. (1980). « Que signifient les erreurs des apprenants? » Langage, $n^{\circ}$ 57, 1980. (Traduction française de « The Significance of Learners Errors ", Review of Applied Linguistics, 1967.

Cuq, J.-P. (Dir.) (2006). Dictionnaire de français langue étrangère et seconde, Paris : CLE International.

Dabène, L. (1992). « Le développement de la conscience métalinguistique : un objectif commun pour l'enseignement de la langue maternelle et des langues étrangères ». Repères, $\mathrm{n}^{\circ} 6, \mathrm{p} .13-21$

Guide du formateur d'enseignants en didactique convergente, (français/arabe), Organisation internationale de la francophonie, 2009. Consulté en janvier 2014. www.lewebpedagogique.com/ oif.

Marquilló Larruy, M. (2006). L’interprétation des erreurs. Paris : CLE international.

Miled, M. (2010 a). Quelle convergence didactique du français et de l'arabe dans le contexte arabophone? Actes du séminaire sur « l'enseignement du français dans le monde arabe ». Paris : CIEP.

Roulet, E. (1980). Langue maternelle et langues secondes, vers une pédagogie intégrée. Paris : HatierCrédif.

Souilah, E. (2014). « Le cycle préparatoire de l'école de base en Tunisie : la difficile adaptation des approches au contexte d'enseignement du français » in Babault, S. et al. (dir.) Actes du colloque 
international « Contexte global, contextes locaux, tensions, convergences, enjeux en didactique des

langues ». Paris : FIPF, p. 98-111.

Taleb-Ibrahimi, K. (2010). Actes du séminaire 7-8 décembre : l'enseignement du français dans les pays de langue arabe. Paris : CIEP.

Vygotsky, L. (1934). Pensée et langage. Paris : La Dispute.

\section{NOTES}

1. Le cas de l'approche par les compétences adoptée par la Tunisie et l'Algérie.

2. Dans le monde arabophone, le français langue seconde est enseigné en Tunisie, en Algérie, au Maroc et au Liban.

3. La didactique convergente, la didactique intégrée, la bivalence sont «trois appellations [...] attestées. » (OIF : 10).

4. «On appelle langue première d'un individu celle qu'il a acquise [...] au moment du développement de sa capacité du langage.» (Cuq, $2006: 152)$

\section{RÉSUMÉS}

Cet article traite la question de l'adaptation de la didactique convergente au contexte arabophone. Il s'agit d'une démarche d'enseignement de la langue conçue en Europe, mais qui peut être porteuse de solutions aux problèmes d'apprentissage du français dans le monde entier. Elle est susceptible d'adaptation à des contextes différents car elle s'appuie sur le potentiel cognitif de l'apprenant et sur sa langue maternelle. Dans l'espoir de remédier aux problèmes de l'enseignement-apprentissage de la langue française dans le contexte arabophone, un travail d'adaptation de la didactique intégrée a été effectué. Il a donné lieu à l'élaboration d'un guide pour la formation des enseignants en didactique convergente / français-arabe. Or l'examen de ce guide montre que pour faire face aux difficultés liées à la particularité et à la complexité sociopsycholinguistique de ce contexte, le travail d'adaptation de cette approche a engendré une version de la didactique convergente en porte-à-faux avec la version initiale.

This article deals with the accommodation of the integrated/convergent didactics to the Arabic context. It is a language teaching procedure devised in the north but which seems to bring up solutions to the French learning problems all over the world. Since it is built on the cognitive potential of the learner along with his mother tongue which is more likely to adaptation to different context. Hoping to remedy the teaching, the adaptation of this procedure has been carried out. This has led to the elaboration of a guide for training teachers of convergent didactics / French-Arabic. However on closer inspection of this guide, we discover that in order to face the difficulties pertaining to the particularity and the sociopsycholinguistic complexity of this context because of the Arabic language status and its variety, the adaptation work has led to a version of didactics at odds with the initial version. 
INDEX

Mots-clés : didactique convergente, français langue seconde, analyse contrastive, contextualisation, arabe

Keywords : integrated learning, French as a second language, contrastive analysis, Arabic, contextualization

\section{AUTEUR}

\section{EMNA SOUILAH}

Docteure en didactique des langues des textes et des cultures, enseignante-chercheuse à l’Université de la Manouba - Tunis. 